\title{
Investigation of turbulent boundary layer flows with adverse pressure gradient by means of 3D Lagrangian particle tracking with Shake-The-Box
}

\author{
M. Novara ${ }^{1 *}$, D. Schanz ${ }^{1}$, R. Geisler ${ }^{1}$, J. Agocs ${ }^{1}$, F. Eich ${ }^{2}$, M. Bross ${ }^{2}$, C. J. \\ Kähler $^{2}$, A. Schröder ${ }^{1}$ \\ ${ }^{1}$ German Aerospace Center (DLR), Inst. of Aerodynamics and Flow Technology, Göttingen, Germany \\ ${ }^{2}$ Universität der Bundeswehr, Inst. of Fluid Mechanics and Aerodynamics, München, Germany \\ *matteo.novara@dlr.de
}

\begin{abstract}
A large-scale 3D Lagrangian particle tracking (LPT) investigation of a turbulent boundary layer (TBL) flow developing across different pressure gradient regions is presented in this study. Three high-speed multi-camera imaging systems, LED illumination and helium-filled soap bubbles (HFSB) tracers have been adopted to produce time-resolved sequences of particle images over a large volume encompassing approximately $3 \mathrm{~m}$ in the streamwise direction, $0.8 \mathrm{~m}$ in the spanwise direction and $0.25 \mathrm{~m}$ in the wall-normal direction. Individual tracers have been reconstructed and tracked within the imaged volume by means of the Shake-The-Box algorithm (STB, Schanz et al. (2016)); the FlowFit data assimilation algorithm (Gesemann et al. (2016)) has been used to evaluate the spatial velocity gradients and to interpolate the scattered LPT results onto a regular grid. Thanks to the large size of the investigated volume and to the time-resolved nature of the recorded images, the entire spatial extent of the large-scale coherent motions within the logarithmic region of the TBL (i.e. superstructures) could be captured and their dynamics investigated during their development over several boundary layer thickness in the streamwise direction, from the zero pressure gradient region (ZPG) to the adverse pressure gradient region (APG). Two free-stream velocities were investigated, namely 7 and $14 \mathrm{~m} / \mathrm{s}$, corresponding to $R e_{\tau} \approx 3,000$ and 5,000 respectively.

The results confirm the location and scale of the elongated high- and low-momentum structures in the logarithmic region, as well as their meandering in the spanwise direction. Two-point correlation statistics show that the width and spacing of the superstructures are not affected by the transition from the ZPG to the APG region. The analysis of the instantaneous flow realizations from both a Lagrangian and Eulerian perspective indicates the presence of significant fluid particle elements exchange across the interfaces of the large-scale structures.
\end{abstract}

\section{Introduction}

The understanding of turbulent boundary layers subjected to an adverse pressure gradient (APG), particularly at high Reynolds numbers, is of high relevance for many aerodynamic applications ranging from lift and thrust generation to wind energy harvesting.

Unlike for the zero pressure gradient case (ZPG), a general consensus about a scaling framework (which could largely benefit the development of near-wall models and wall-functions for RANS numerical simulations - Knopp et al. (2015)) is still missing. Of great interest is also the understanding of the flow separation mechanism, in particular regarding the role played by localized near-wall reverse flow events that might represent the nucleus for the generation of the macroscopic flow separation (Bross et al. (2019)). Furthermore, the interaction between large-scale coherent motions within the logarithmic region of the turbulent boundary layer (i.e. superstructures, Adrian (2007), Hutchins and Marusic (2007), Marusic et al. (2010)) and the near-wall flow structures in the viscous and buffer layers has been receiving increasing attention (Bross et al. (2019)).

These considerations motivate the need to perform accurate flow measurements in the near-wall region at relevant Reynolds numbers. The three-dimensional nature of the flow structures (e.g. strong correlation 
between near-wall reverse flow events and large span-wise velocity component, Diaz-Daniel et al. (2017)) calls for the adoption of multi-point volumetric techniques allowing for the evaluation of spatial correlations and structural analysis. Based on these requirements, 3D Lagrangian particle tracking (LPT) approaches appear to be ideal candidates when the choice of the measurement technique is concerned. In particular, the Shake-The-Box algorithm (STB, Schanz et al. (2016)) enables 3D LPT at relatively high seeding densities (Huhn et al. (2017) among others), comparable and exceeding those typically employed for cross-correlation based techniques. Unlike for cross-correlation-based methods, particle tracking approaches can deliver reliable measurements in close proximity of interfaces, walls and strong shear layers (Kähler et al. (2012a), Kähler et al. (2012b), Cierpka et al. (2013)).

In the present study the attention is directed at the investigation of the large-scale coherent motions within the logarithmic region of the TBL (i.e. superstructures, Bross et al. (2019) and Marusic et al. (2010) among others); the main questions concern the influence of the pressure gradient on the occurrence and scaling of the large-scale structures (LSS), the interaction of these structures with the small-scales close to the wall and local backflow events, and whether or not an exchange of fluid elements occurs across the interface of the large-scale structures

A large-scale 3D time-resolved investigation has been performed with STB making use of twelve highspeed cameras, helium-filled soap bubbles (HFSB) and LED illumination to cover the flow development over nearly three meters along the streamwise direction (from a zero-pressure gradient region and into an APG region). The experimental campaign took place in the Atmospheric Wind Tunnel (AWM) at the University of Armed Forces (Munich) over the same model designed within the scope of the DLR project VICTORIA (previous results from this project can be found in Schröder et al. (2018)). The experimental setups are presented here together with results in terms of analysis of the instantaneous 3D particle tracks and flow structures (identified by interpolating the scattered LPT results to a regular grid by means of the FlowFit algorithm - Gesemann et al. (2016)), and flow statistics (obtained via ensemble-averaging of LPT data).
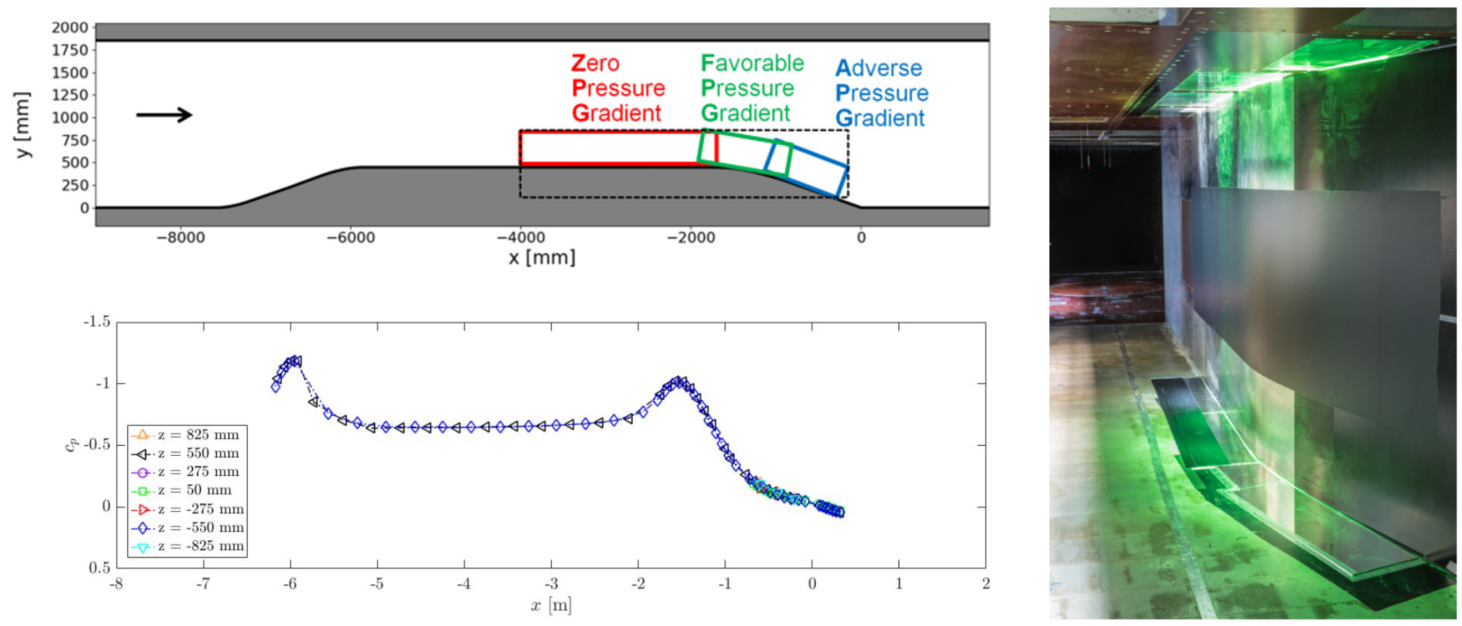

Figure 1: Left: sketch of the model (top) and pressure distribution measured at the AWM facility (bottom). Right: model installed in the AWM test section and LED illumination.

\section{Experimental setup}

A sketch of the wind-tunnel model installed on the side wall of the AWM facility test section is shown in figure 1-top-left; the closed test section of the open circuit facility is $22 \mathrm{~m}$ long and has a cross-sectional area of $1.8 \times 1.8 \mathrm{~m}^{2}$.

In order to qualify the model, the pressure distribution at the wall was measured; results are shown in figure 1-bottom-left. After a canonical ZPG region developing over a $4 \mathrm{~m}$ long flat plate, two curvilinear deflections cause a small favorable pressure gradient (FPG) followed by an APG region over the $763 \mathrm{~mm}$ flat plate inclined by $18^{\circ}$.

A system of twelve high-speed cameras was installed outside of the tunnel, opposite to the model, figure 2 -left. The cameras are organized into three imaging systems; the overlapping volumetric field-of-views 

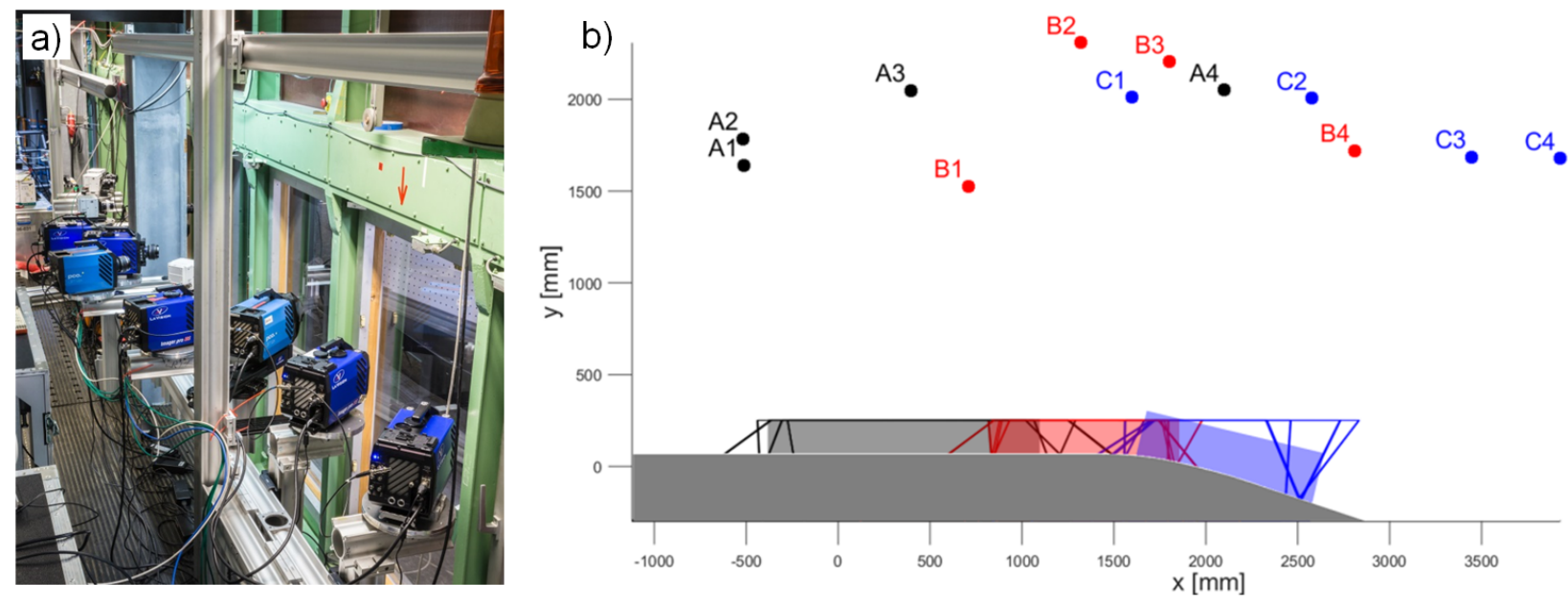

Figure 2: Left: twelve high-speed cameras installed outside the AWM test-section. Right: sketch of the model and imaging system; the three camera systems $(A, B, C)$ and the relative imaged volumes are indicated in black, red and blue respectively. Reproduced from Schanz et al. (2019).

(figure 2-right) cover a continuous volume from the middle of the ZPG plate to the APG region $(\approx 2.90 \mathrm{~m}$ streamwise, $0.8 \mathrm{~m}$ span-wise and $0.25 \mathrm{~m}$ wall-normal). The cameras were operated at a constant repetition rate of $1 \mathrm{kHz}$ and recorded continuous data for 1382 images; several statistically independent individual runs were acquired in order to produce the flow statistics. The most upstream camera system $(A)$ consisted of four VEO $4 \mathrm{~K} \mathrm{~L}$ cameras, which provide a $4096 \times 2160$ pixels resolution at $1 \mathrm{kHz}$. Due to the high spatial resolution and the elongated shape of the sensors, this system was able to capture the full ZPG-region at a length of $1.80 \mathrm{~m}$, using $f=35 \mathrm{~mm}$ Carl Zeiss Distagon lenses, mounted in Scheimpflug-adapters. The two subsequent systems $(B$ and $C)$ each consisted of four PCO DiMax cameras $(2016 \times 2016$ pixels $)$ and each covered around $1 \mathrm{~m}$ in streamwise direction, being equipped with $f=50 \mathrm{~mm}$ Carl Zeiss Planar lenses. The magnification ranges between 2.0 to $2.5 \mathrm{px} / \mathrm{mm}$ for the whole system.

The wall-normal extent of the recorded volume is defined by the illumination, consisting of 10 highpower LED arrays (Hardsoft ILM 501CG, Stasicki et al. (2017)), installed above window inserts on the wind tunnel roof; mirrors at the wind tunnel floor provided back-reflection, figure 1 1 -right. Two different free stream velocities were considered, $U_{\infty} \approx 7$ and $14 \mathrm{~m} / \mathrm{s}$. At the lowest velocity, particle shifts of around $19 p x$ were found in the free stream. In order to limit the shift for the higher velocity, a double illumination scheme was applied, motivated by its successful application on double frame cameras for the investigation of high-speed flows (Novara et al. (2019)). While the cameras retained the rate of $1 \mathrm{kHz}$, the LEDs were operated at $2 \mathrm{kHz}$, thus imaging each particle twice per camera image.

Seeding of the flow was realized by several arrays of HFSB-nozzles (250 nozzles in total). For the lower velocity, most of the seeding rakes (200 nozzles) were installed close to the contraction before the test section. While ensuring a high seeding concentration, a downside of this position is an interaction of the rakes with the incoming boundary layer that might persist in the measurement region. For the run at higher Reynolds number the seeding system was moved further upstream; the bubbles had to pass through several layers of meshes, minimizing any flow disturbances, but also reducing the number of bubbles reaching the measurement volume. In both cases, 50 additional nozzles were installed in a cabinet close to the contraction region, seeding the near-wall parts of the boundary layer through a thin slit in the wind tunnel wall.

For the $U_{\infty} \approx 7 \mathrm{~m} / \mathrm{s}$ case 24 runs of 1382 images each were recorded in single illumination mode. The LEDs were operated only in short bursts of $40 \mu \mathrm{s}$ per image ( $4 \%$ duty cycle, at $45 \mathrm{~A}$ power), in order to avoid any motion blurring for the fastest particles. At the higher velocity the seeding concentration is reduced both due to the increased mass flow and the different position of the main seeding system; however, the particle image density is doubled due to the two light pulses. For $U_{\infty} \approx 14 \mathrm{~m} / \mathrm{s} 68$ runs of 1382 recordings (corresponding to 2764 time-steps) were recorded. 


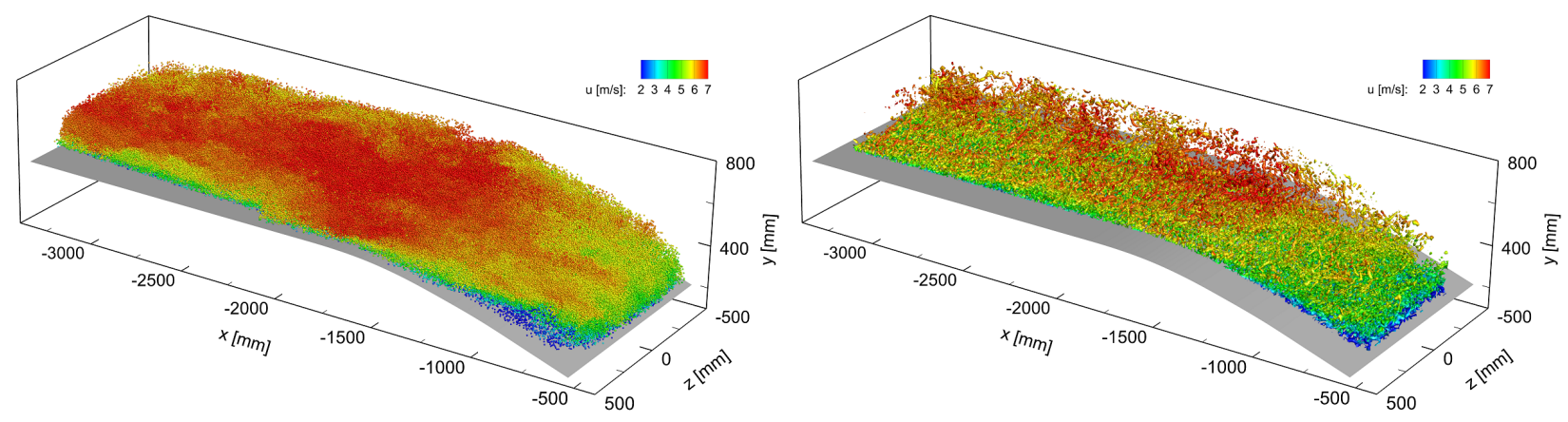

Figure 3: Instantaneous result from STB (left) and FlowFit (right) for the $7 \mathrm{~m} / \mathrm{s}$ case. Left: $\approx 600,000$ tracked particles color-coded with streamwise velocity. Right: iso-surface of Q-criterion at $20001 / \mathrm{s}^{2}$.

\section{Results}

The STB algorithm was adapted to seamlessly triangulate (via Iterative Particle Reconstruction, IPR Wieneke (2012)) and track the bubbles over the whole volume (across the three imaging systems) and to cope with the double-exposed recordings for $U_{\infty}=14 \mathrm{~m} / \mathrm{s}$. A detailed description of the processing strategy and parameters can be found in Schanz et al. (2019). The particle image density ranged between 0.04 and 0.06 particles-per-pixel ( $p p p$ ); a number of approximately 620,000 and 200,000 tracked particles was found within the complete measurement volume for the single- and double-exposed recordings respectively. Particle positions along the tracks were filtered by the TrackFit spline-interpolation scheme (Gesemann et al. (2016)) in order to deliver continuous position, velocity and acceleration measurements.

An example of an instantaneous result from TR-STB is presented in figure 3 -left for the $7 \mathrm{~m} / \mathrm{s}$ case; approximately 600,000 tracks are color-coded by the stream-wise velocity component. The application of the FlowFit data assimilation algorithm (Gesemann et al. (2016)) allows for the identification of instantaneous 3D flow structures in the TBL; figure 3-right shows an example of instantaneous iso-surfaces of Q-criterion color-coded by the stream-wise velocity component. Medium-scale structures in the outer part of the boundary layer appear to be well resolved; as a consequence of the large measurement domain and the size of the HFSB tracers ( $300 \mu m$ diameter) an under sampling of the flow gradients in the near-wall region is expected.

The scattered results from LPT can be used to evaluate flow statistics by means of an ensemble averaging approach. In Figure figure 4. left the mean streamwise velocity component for the $14 \mathrm{~m} / \mathrm{s}$ is presented; 3D bins, of $10 \times 10 \times 10 \mathrm{~mm}^{3}$ size, were employed and approximately 100,000 samples per bin used to evaluate the flow statistics. In order to characterized the TBL, a $2 \mathrm{D}$ ensemble averaging analysis was carried out making use of bins encompassing the whole spanwise dimension $(Z), 5 \mathrm{~mm}$ along the streamwise direction
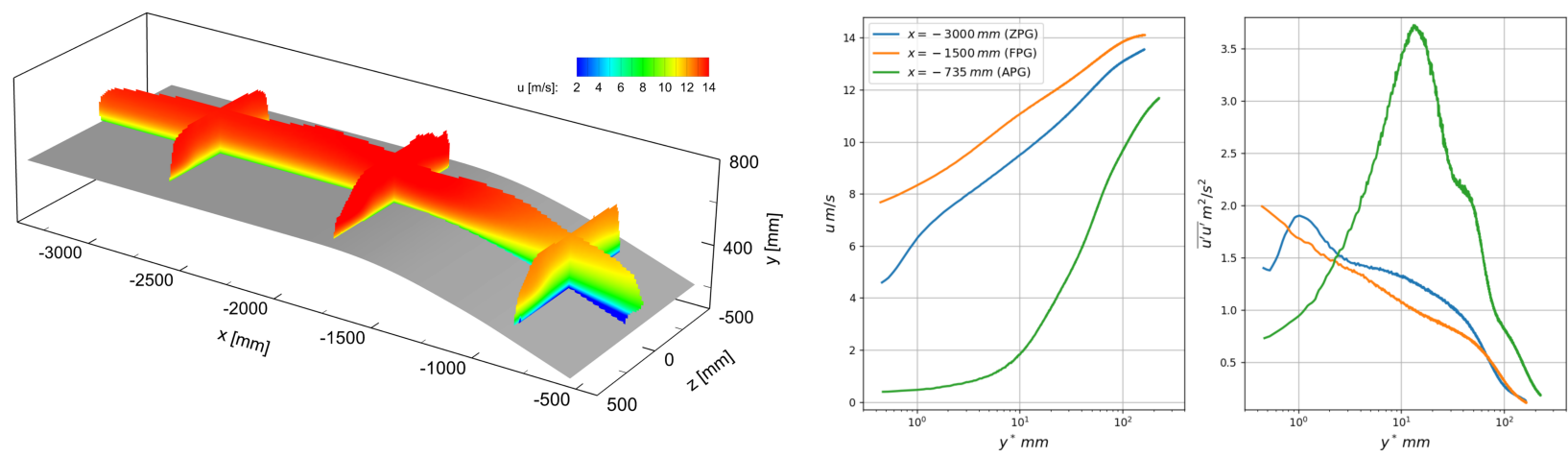

Figure 4: Results from ensemble averaging of STB results for the $14 \mathrm{~m} / \mathrm{s}$ case. Left: mean streamwise velocity component $\left(10 \times 10 \times 10 \mathrm{~mm}^{3}\right.$ bins). Right: wall-normal streamwise velocity and Reynolds stresses component from $2 \mathrm{D}$ ensemble averaging analysis $\left(5 \times 0.1 \mathrm{~mm}^{2}\right.$ bins in $X Y$ plane $)$ in ZPG, FPG and APG. 

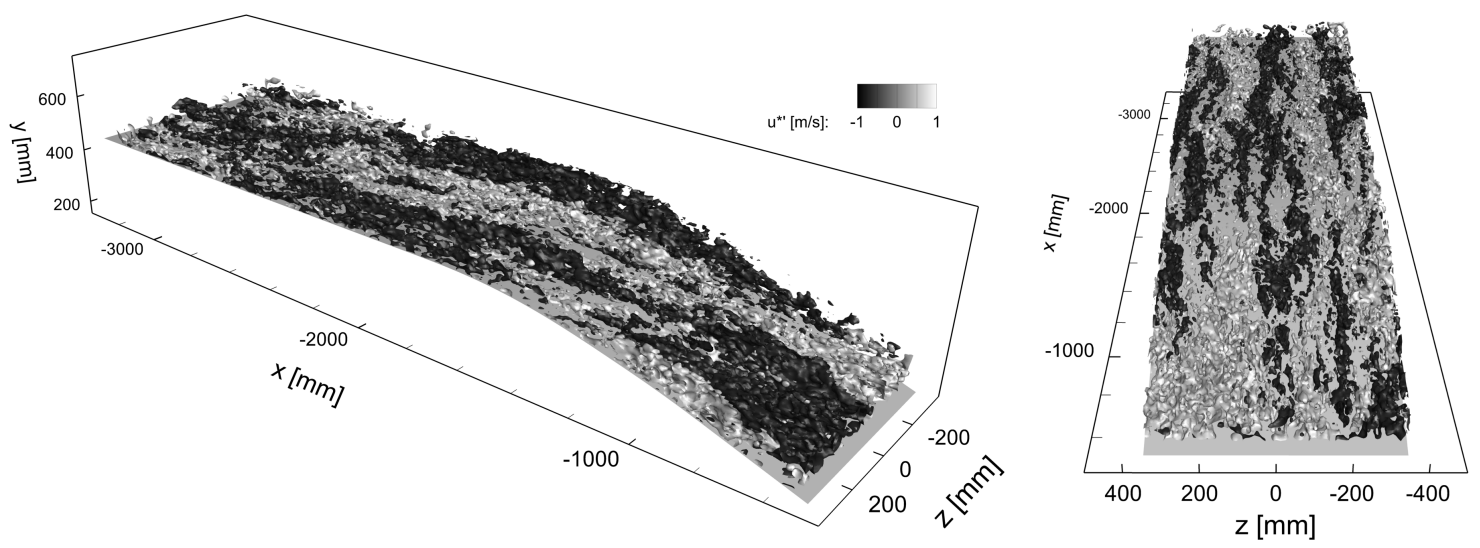

Figure 5: Two views of instantaneous large-scale structures in the logarithmic layer for the $14 \mathrm{~m} / \mathrm{s}$ case; highand low-momentum structures visualized by iso-surfaces of positive and negative wall-parallel fluctuation velocity component $\left(u^{*^{\prime}}= \pm 1 \mathrm{~m} / \mathrm{s}\right)$.

$(X)$ and $0.1 \mathrm{~mm}$ along the ZPG-wall-normal direction $(Y)$; approximately 35,000 samples per bin were found for the $14 \mathrm{~m} / \mathrm{s}$ case. Mean streamwise velocity and Reynolds stresses profiles along the local wall-normal direction $\left(y^{*}\right)$ are presented in figure 4-right for three locations in the ZPG, FPG and APG respectively (blue, orange and green curves). The slight acceleration of the flow caused by the FPG at $X \approx-1500 \mathrm{~mm}$ can be observed, followed by the deceleration experienced when entering the APG region. The streamwise component of the Reynolds stresses shows that, despite the large size of the measurement volume, the first peak of the fluctuations can be resolved in the ZPG region. As expected, in the APG region the $u^{\prime} u^{\prime}$ peak is broader and located further away from the wall. The Clauser chart analysis (Clauser (1956)) of the incoming boundary layer (ZPG region, $X=-2650 \mathrm{~mm}$ ) allowed for the evaluation of the viscous unit size $\left(l^{+} \approx 60\right.$ and $30 \mu \mathrm{m}$ for 7 and $14 \mathrm{~m} / \mathrm{s}$ respectively) and the friction velocity $\left(u_{\tau} \approx 0.26\right.$ and $0.5 \mathrm{~m} / \mathrm{s}$ for 7 and $14 \mathrm{~m} / \mathrm{s}$ respectively). The incoming boundary layer thickness $\delta_{99}$ was found to be approximately $170 \mathrm{~mm}$ for the $14 \mathrm{~m} / \mathrm{s}$ case. The Reynolds numbers based on the friction velocity $\left(R e_{\tau}\right)$ was estimated at approximately 3400 and 5400 for the lower and higher free-stream velocity respectively. Due to the disturbance to the outer flow caused by the seeding rakes installed before the test section for the $7 \mathrm{~m} / \mathrm{s}$ case, the TBL profile was not canonical, and the TBL thickness could not be estimated accurately. On the other hand, a comparison of the TBL profiles with numerical and experimental results from the literature (Sillero et al. (2013), Samie et al. (2018)) confirmed that, for both free-stream velocities, the TBL profile behaves as expected over the whole logarithmic region, where the superstructures are located.

Two views of an instantaneous realization of the FlowFit result are presented in figure 5, where isosurfaces of wall-parallel velocity fluctuations $\left(u^{*^{\prime}}\right)$ in the logarithmic layer are shown for the $14 \mathrm{~m} / \mathrm{s}$ case. As reported by Bross et al. (2019) among others, the high- and low-momentum regions (in white and black respectively in figure 5) are elongated in the streamwise direction and can extend up to several boundary layer thicknesses when instantaneous flow fields are considered (up to $10-20 \cdot \delta_{99}$ ). The results shown in figure 5 also confirm the meandering of the superstructures in the spanwise direction. At high Reynolds numbers, these superstructures strongly contribute to the second peak in the streamwise velocity fluctuations.

The average size, orientation and spanwise spacing of large-scale coherent structures in the TBL can be estimated by analyzing the 3D two-point spatial cross-correlation coefficient of the streamwise velocity fluctuations $R_{u^{\prime} u^{\prime}}$ (Chen (2019)). An ensemble averaging approach was employed to evaluate two-point cross-correlation maps from the Lagrangian data offered by STB. Three locations along the streamwise direction (i.e. ZPG, FPG and APG region) were selected for the reference points and the two-point correlation coefficient was evaluated over the entire 3D field at different locations along the wall-normal direction. In order to investigate the influence of the pressure gradient on the average structural topology, the relevant length scales calculated from the analysis of the two-point correlation coefficient maps, are shown in figure 6 as a function of the wall-normal position.

The length scale in the streamwise direction corresponds to the total length of the correlation up to a threshold value $\left(L_{1}\right)$ or to the length of the correlation in a section plane parallel to the wall $\left(L_{2}\right)$; due to the inclination of the structures relative to the wall, $L_{1}$ is always larger than $L_{2}$. The spanwise separation $\Lambda_{2}$ is the distance between the minima of the correlation coefficient along the spanwise direction $Z$. Finally, the 
a)

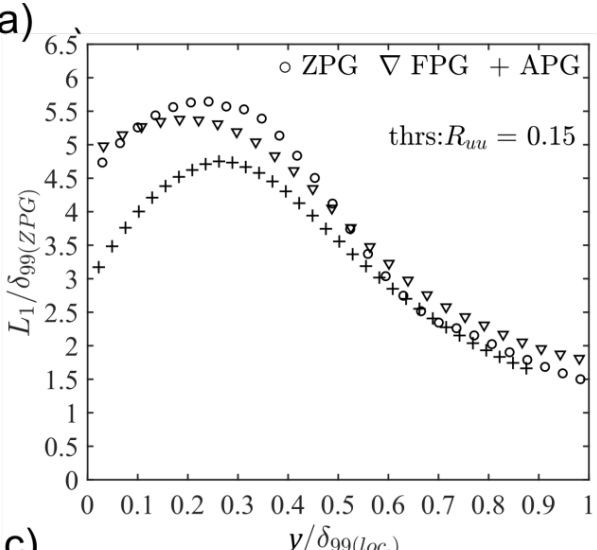

c)

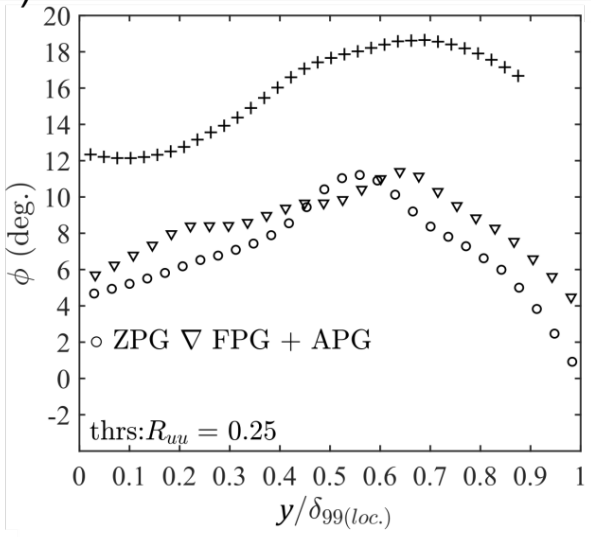

b)
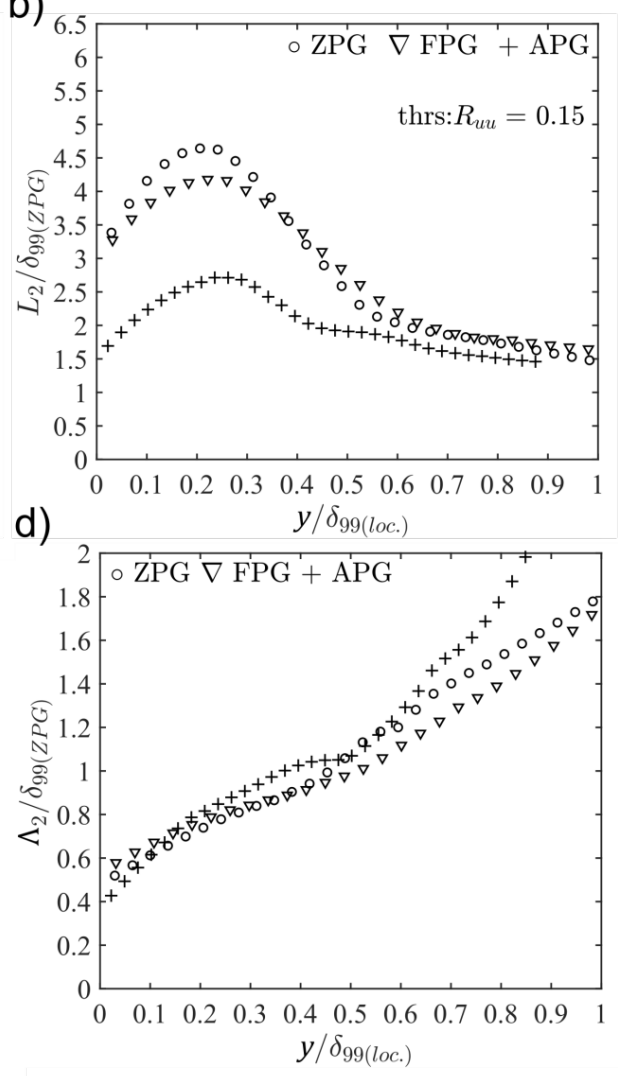

Figure 6: Length scales $L_{1}$ (a) and $L_{2}$ (b) calculated from two-point correlations $R_{u^{\prime} u^{\prime}}$. Relative structure inclination angle (c) and spanwise distance of the large-scale structures calculated from the minima of the $R_{u^{\prime} u^{\prime}}$ correlation (d).

relative tilt angle is calculated by approximating the positive $R_{u^{\prime} u^{\prime}}$ correlation contour at a given threshold with an ellipse; the angle $\phi$ corresponds to the angle of the major semi-axis relative to the wall.

The length scales $L_{1}$ and $L_{2}$ are normalized using the boundary layer thickness in the ZPG $\left(\delta_{99, Z P G}\right)$ in order to allow comparing the structures in the different pressure gradient regions despite the change in the local boundary layer thickness. For both $L_{1}$ (figure 6a) and $L_{2}$ (figure 6b), there is almost no difference between the ZPG and FPG regions, where the maximum values are located at approximately $y / \delta_{99, l o c}=0.25$. In contrast, $L_{1}$ and $L_{2}$ are significantly smaller at the APG position if they are scaled with $\delta_{99 . Z P G}$; the shortening of the structures is due to the strong deceleration of the flow caused by the APG.

This behavior is also visible in the representation of the relative angle of inclination in figure $6 \mathrm{c}$. For the ZPG and FPG regions, the maximum relative angle of inclination is approximately $10^{\circ}$, while the inclination angle in the APG is about twice as large for each distance from the wall. Interestingly, the structure distance $\Lambda_{2}$ shown in figure $6 \mathrm{~d}$ is similar for all pressure gradient cases, particularly for the wall positions $y / \delta_{99, l o c}<$ 0.5 . It can therefore be stated that the structural pattern in the spanwise direction does not physically change under the influence of an APG compared to the ZPG and FPG regions,

The time-resolved 3D fields obtained from the combined STB and FlowFit approaches offer the unique opportunity to investigate the dynamic behavior of the superstructures travelling across the different pressure regions over the wind-tunnel model. The results presented in figure 7 show the evolution of a portion of the flow field (approximately $200 \mathrm{~mm}$ long in the streamwise direction) followed as it is advected downstream with a fixed local average flow velocity of $\approx 6 \mathrm{~m} / \mathrm{s}$ across the ZPG, FPG and APG regions; the location of the flow field patch is shown in yellow in the sketches in figure 7-top. The superstructures are shown with isosurfaces of $u^{*^{\prime}}$, while instantaneous vortical structures are identified by iso-surfaces of vortex identification criterion $Q$, color-coded by streamwise velocity component; by observing the temporal evolution of the flow structures, it appears that streets of vortices ride on top of low-momentum structures while maintaining a rather stable spatial organization. 

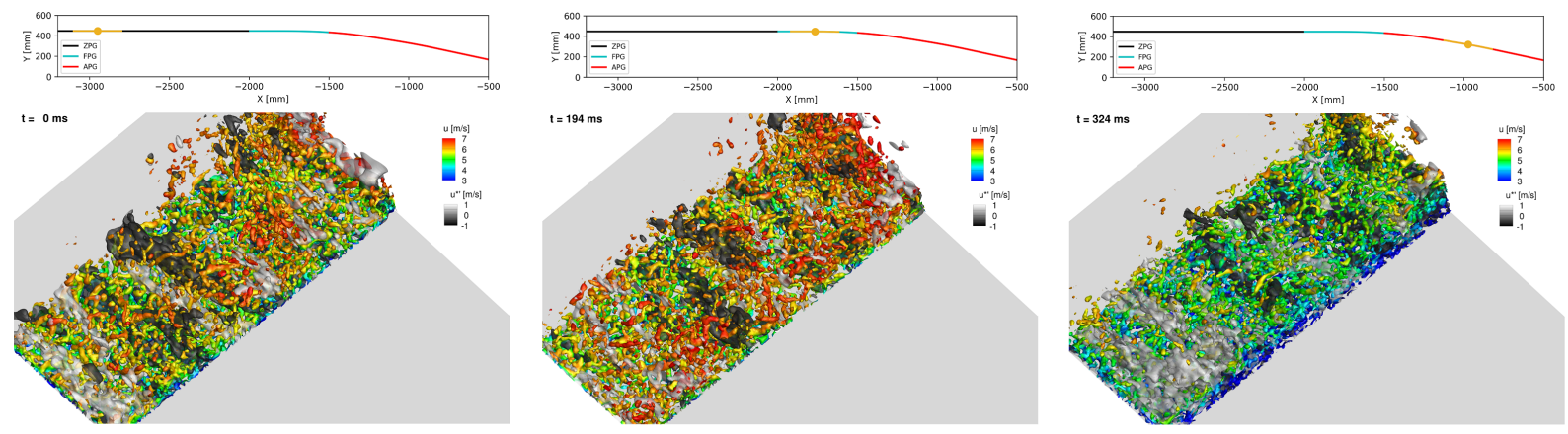

Figure 7: Instantaneous LSSs (iso-surfaces of $u^{*^{\prime}}= \pm 0.6 \mathrm{~m} / \mathrm{s}$ ) and vortical structures (iso-surface of $Q=2,5001 / \mathrm{s}^{2}$ ) color-coded by streamwise velocity component for the $7 \mathrm{~m} / \mathrm{s}$ case. Same flow patch advected downstream shown in the ZPG (left), FPG (center) and APG (right) region. Location of the flow patch (in yellow) with respect to the pressure gradient regions (black, blue and red for ZPG, FPG and APG respectively) shown on top.

The Eulerian coherence of the turbulent superstructures, inferred by the visualization of time-resolved sequences, can be quantified by evaluating the two-point space-time correlation coefficient of the streamwise velocity fluctuations which provides an indication about the memory of the process (Chen (2019)). A fixed reference point is chosen in the ZPG region (at different wall-normal locations, namely $y^{+} \approx 1,000,2,600$ and 5,300) and the cross-correlation coefficient is computed for a series of time separations, namely 0,100 , 200 and $300 \mathrm{~ms}$. The results, relative to the $14 \mathrm{~m} / \mathrm{s}$ case, are presented in terms of positive and negative iso-surfaces of $R_{u^{\prime} u^{\prime}}$ in figure 8 ; the elongated shape of the correlation coefficient iso-surfaces reflects the shape of the superstructures, while the stronger correlation values attained in proximity of $0.5 \cdot \delta_{99}$ (figure 8 -middle) confirm the location of the superstructures in the upper logarithmic layer.
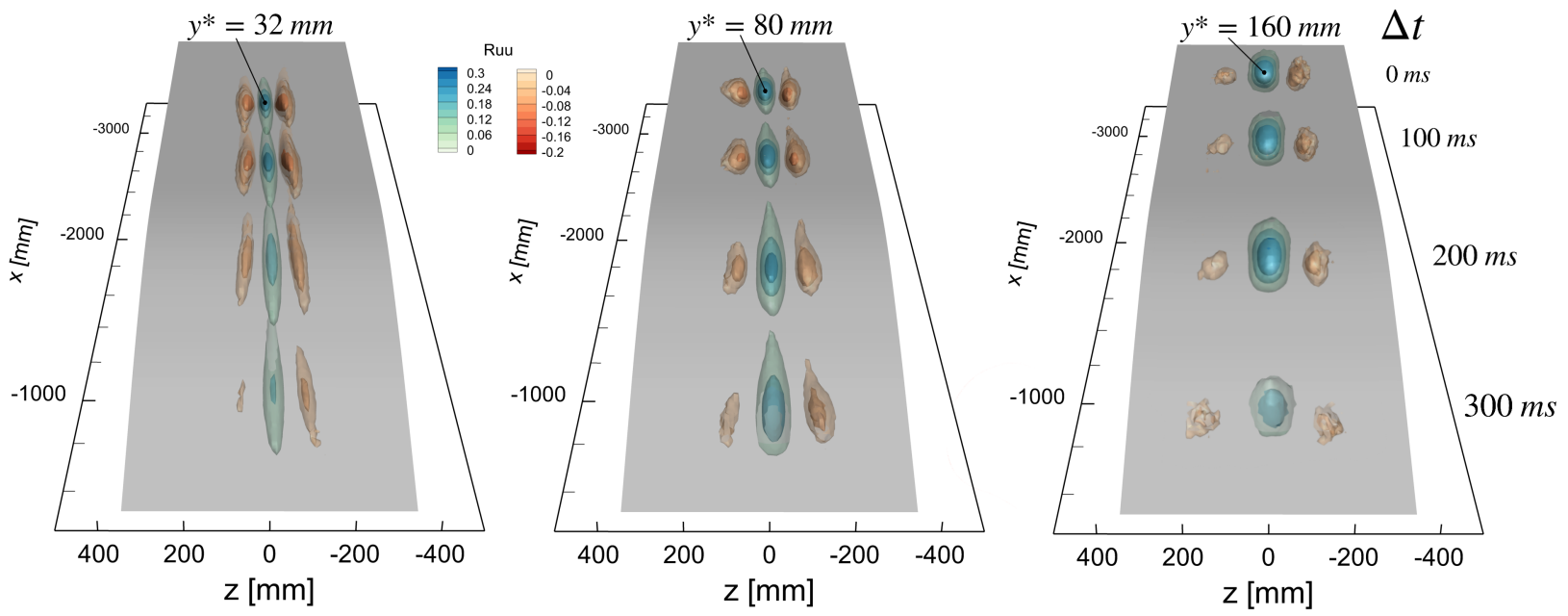

Figure 8: Iso-surfaces of two-point space-time correlation coefficient for the $14 \mathrm{~m} / \mathrm{s}$ case for different locations of the reference point (black dot) along the wall-normal direction in the ZPG $(X=-2420 \mathrm{~mm})$ and for different time separations $(\Delta t)$. Positive values of $R_{u^{\prime} u^{\prime}}=0.12 / 0.2 / 0.3$ in blue and negative ones in orange $\left(R_{u^{\prime} u^{\prime}}=-0.055 /-0.07 /-0.1\right)$.

In order to assess the presence of fluid element exchange across the interfaces of the superstructures, the dual nature of the STB and FlowFit results (i.e. Lagrangian and Eulerian views of the flow) can be taken advantage of. When a time-resolved sequence of realizations is considered, similarly to what is presented in figure 7, iso-surfaces of $u^{*^{\prime}}$ obtained from the FlowFit interpolation can be visualized for the low- and high- 

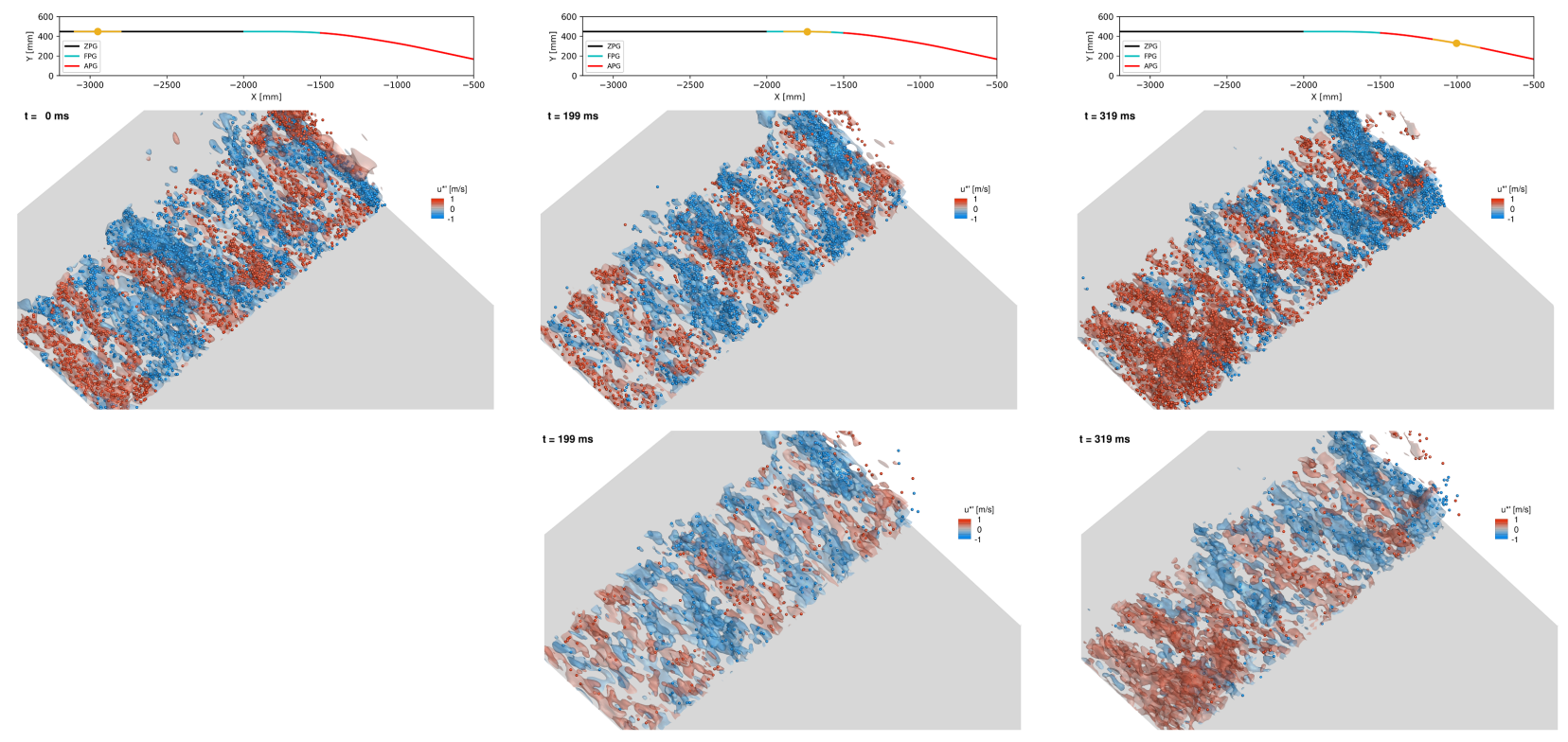

Figure 9: Instantaneous LSSs (iso-surfaces of $u^{*^{\prime}}= \pm 0.6 \mathrm{~m} / \mathrm{s}$ ) and particles color-coded by wall-parallel velocity fluctuation component for the $7 \mathrm{~m} / \mathrm{s}$ case. Same flow patch advected downstream shown in the ZPG (left), FPG (center) and APG (right) region. Particles within the LSSs showed as spherical markers: top: all instantaneous particles within the superstructures, bottom: subset of particles already present at $t=0 \mathrm{~ms}$.

momentum superstructures (in blue and red in figure 9 respectively) for the same portion of the flow field imaged at different instants across its path from the ZPG to the APG region. At the same time, individual particle tracers from STB can be visualized by spherical markers color-coded by $u^{*^{\prime}}$ values; only particles within the superstructures are shown in figure 9. When all instantaneous particles exhibiting a large value of wall-parallel turbulent fluctuation are considered, figure 9-top, it can be observed, as expected, that the instantaneous superstructures are populated by a rather homogeneous number of individual tracers. On the other hand, when the plotted tracers are limited to those already present at the beginning of the time sequence (i.e. $t=0 \mathrm{~ms}$, figure 9 -top-left), a progressively decreasing number of particles is found within the structures as they are advected downstream (figure 9-bottom).

This visualization allows to conclude that, despite looking quite stable when moving downstream during the observation time of the STB recording sequence in the Eulerian frame of reference (see persisting vortical and streaky flow structures in figure 77, the turbulent superstructures are sustained by particles belonging to different individual tracks, therefore confirming the presence of fluid particle elements exchange across the interfaces of the LSSs.

A similar conclusion can be drawn when complete individual tracks crossing a high- (figure 10-left) or low-momentum (figure 10-right) superstructure at a given time instant (larger blue and red markers in figure 10 are visualized, color-coded by the wall-normal velocity fluctuation value. The locations marked in gray along the trajectory indicate that the particle is located outside of a superstructure, while portions of the same track colored in red and blue show how the particle can enter different types of structures during its travel across the investigated domain.

Figure 10 also shows that particles that are spatially relatively close to each other at one particular time instant occupy significantly different regions of space when the wall-normal $\left(y^{*}\right)$ and spanwise $(Z)$ directions are considered at different time instants (i.e. streamwise locations). This dispersion can be quantified by performing a single-particle dispersion analysis (Zhang and Xiao (2018)) where the quadratic distances along $y^{*}$ and $Z$ for each position of a particle along a track are computed with respect to the particle position at a reference time $(\tau=0 \mathrm{~ms})$. When a statistical analysis is performed by averaging the dispersion results over multiple tracks, single-particle dispersion curves as presented in figure 11 can be obtained. For each track, the reference time was chosen as the time at which the particle passed close to a specific location (i.e. reference point) within the FPG; the choice of the FPG region was dictated by the fact that this position, being the FPG located approximately at the center of the FOV along the streamwise direction, would allow 

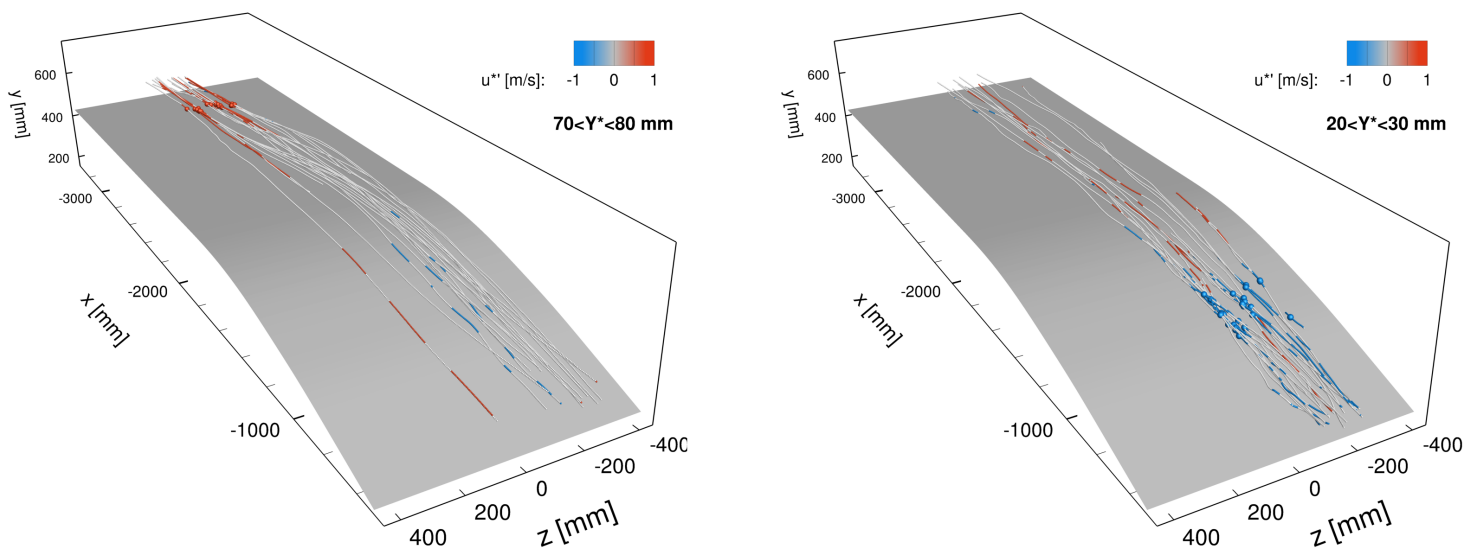

Figure 10: Selection of complete tracks color-coded by wall-parallel velocity fluctuation component; particle locations at a chosen time instant where particles belong to a high- (left) or low-momentum (right) superstructures are shown using larger markers.

for the evaluation of both backward $(\tau<0)$ and forward $(\tau>0)$ dispersion.

The dispersion analysis was performed for several reference points distributed along the wall-normal direction up to $y^{*}=\delta_{99, Z P G}$ with a spacing of $20 \mathrm{~mm}$ from each other; all particle tracks crossing an area of $50 \times 10 \mathrm{~mm}^{2}$ in the $X y^{*}$ plane around the reference point over a full time-resolved recording sequence were included in the dispersion analysis.
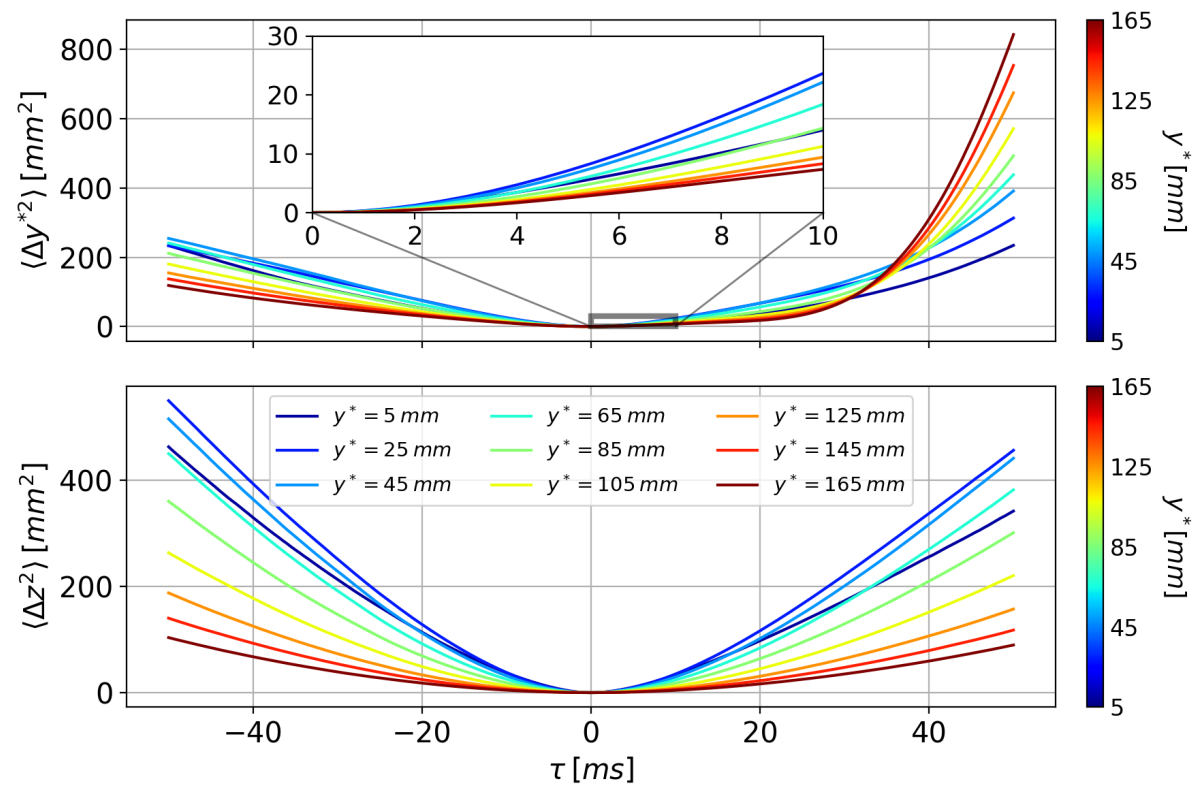

Figure 11: Backward and forward single-particle dispersion in the wall-normal (top) and spanwise direction (bottom); reference volumes located in the FPG $(x=-1750 \mathrm{~mm})$ at different locations along the wall-normal direction $y^{*}$.

The wall-normal and spanwise dispersion results are shown in figure 11(top and bottom respectively); dispersion curves are color-coded based on the reference point location in the wall-normal direction. Given the location of the reference point at $X=-1750 \mathrm{~mm}$ (FPG), negative values of the time separation $\tau$ indicate results relative to particles coming from the ZPG region, while positive $\tau$ values show the particles dispersion as the tracers enter the APG region. Results show that the spanwise dispersion is larger than the wall-normal one for $|\tau|<30 \mathrm{~ms}$; on the other hand, when particles enter the APG region, they exhibit a larger deviation 
in terms of their relative wall-normal distance from the wall. Furthermore, both the wall-normal and the spanwise dispersion attain a maximum value within the logarithmic region for $|\tau|<30 \mathrm{~ms}$, which can be associated to the meandering nature of the superstructures and their inclination in the $X y^{*}$ plane.

\section{Conclusions and outlook}

The adoption of three high-speed multi-camera imaging systems, LED illumination and helium-filled soap bubbles, in combination with the Shake-The-Box 3D LPT algorithm, allowed for the investigation of an unprecedented large-scale volume within a TBL flow crossing three pressure gradient regions (ZPG, FPG and APG). The three-dimensional nature of the experiment enabled capturing large-scale coherent structures in the logarithmic layer (i.e. superstructures) in their entirety; the time-resolved recording sequences made it possible to investigate the flow dynamics.

Visual inspection of the instantaneous flow realizations and the results from two-point spatial correlation statistics confirmed the presence of the large-scale structures and suggested that their spatial organization is not affected by the transition across the pressure gradient regions. The availability of both Lagrangian and Eulerian data allowed to determine that significant fluid elements exchange occurs across the interfaces of the superstructures. Single-particle dispersion analysis of individual tracks confirmed the meandering nature of the LSSs and their increased inclination in the APG region.

Together with the analysis of two-particle dispersion (Salazar and Collins (2009)), spatial clustering algorithms could be applied to further characterize the behavior of the turbulent superstructures in the TBL (Schneide et al. (2018)). Furthermore, conditional ensemble averaging approaches based on the detection of individual sweep $\left(Q_{4}\right)$ and ejection $\left(Q_{2}\right)$ events would allow to investigate the relation between the superstructures and the turbulence production at varying distances from the wall. Furthermore, two-point cross-correlation analysis involving velocity and instantaneous pressure from the FlowFit algorithm will be employed to investigate the role of the pressure fluctuations induced by the large-scale structures for the local and global separation processes at the wall.

\section{Acknowledgements}

The present work has been partially funded by the DFG-project "Analyse turbulenter Grenzschichten mit Druckgradient bei großen Reynolds-Zahlen mit hochauflösenden Vielkameramessverfahren" (grant No. SCHR 1165/3-2 and KA1808/14-2). The wind tunnel model was laid out by Tobias Knopp and installed as part of the DLR project VICTORIA.

\section{References}

Adrian RJ (2007) Hairpin vortex organization in wall turbulence. Physics of Fluids 19:041301

Bross M, Fuchs T, and Kähler CJ (2019) Interaction of coherent flow structures in adverse pressure gradient turbulent boundary layers. Journal of Fluid Mechanics 873:287-321

Chen J (2019) Two-point statistics of coherent structure in turbulent flow. arXiv preprint arXiv:190805422

Cierpka C, Lütke B, and Kähler CJ (2013) Higher order multi-frame particle tracking velocimetry. Experiments in Fluids 54:1-12

Clauser FH (1956) The turbulent boundary layer. in Advances in applied mechanics. volume 4. pages 1-51. Elsevier

Diaz-Daniel C, Laizet S, and Vassilicos JC (2017) Wall shear stress fluctuations: Mixed scaling and their effects on velocity fluctuations in a turbulent boundary layer. Physics of Fluids 29:055102

Gesemann S, Huhn F, Schanz D, and Schröder A (2016) From noisy particle tracks to velocity, acceleration and pressure fields using b-splines and penalties. in 18th international symposium on applications of laser and imaging techniques to fluid mechanics, Lisbon, Portugal. pages 4-7 
Huhn F, Schanz D, Gesemann S, Dierksheide U, van de Meerendonk R, and Schröder A (2017) Large-scale volumetric flow measurement in a pure thermal plume by dense tracking of helium-filled soap bubbles. Experiments in Fluids 58:1-19

Hutchins N and Marusic I (2007) Evidence of very long meandering features in the logarithmic region of turbulent boundary layers. Journal of Fluid Mechanics

Kähler CJ, Scharnowski S, and Cierpka C (2012a) On the resolution limit of digital particle image velocimetry. Experiments in fluids 52:1629-1639

Kähler CJ, Scharnowski S, and Cierpka C (2012b) On the uncertainty of digital piv and ptv near walls. Experiments in fluids 52:1641-1656

Knopp T, Buchmann N, Schanz D, Eisfeld B, Cierpka C, Hain R, Schröder A, and Kähler CJ (2015) Investigation of scaling laws in a turbulent boundary layer flow with adverse pressure gradient using piv. Journal of Turbulence 16:250-272

Marusic I, McKeon BJ, Monkewitz PA, Nagib HM, Smits AJ, and Sreenivasan KR (2010) Wall-bounded turbulent flows at high reynolds numbers: recent advances and key issues. Physics of Fluids 22:065103

Novara M, Schanz D, Geisler R, Gesemann S, Voss C, and Schröder A (2019) Multi-exposed recordings for 3d lagrangian particle tracking with multi-pulse shake-the-box. Experiments in Fluids 60:1-19

Salazar JP and Collins LR (2009) Two-particle dispersion in isotropic turbulent flows. Annual review of fluid mechanics 41:405-432

Samie M, Marusic I, Hutchins N, Fu M, Fan Y, Hultmark M, and Smits A (2018) Fully resolved measurements of turbulent boundary layer flows up tore $\tau=20,000)$. Journal of Fluid Mechanics 851:391-415

Schanz D, Gesemann S, and Schröder A (2016) Shake-the-box: Lagrangian particle tracking at high particle image densities. Experiments in fluids 57:1-27

Schanz D, Schröder A, Novara M, Geisler R, Agocs J, Eich F, Bross M, and Kähler CJ (2019) Large-scale volumetric characterization of a turbulent boundary layer flow. in Proceedings of the 13th International Symposium on Particle Image Velocimetry. 182. pages 251-265. Universität der Bundeswehr München: AtheneForschung

Schneide C, Pandey A, Padberg-Gehle K, and Schumacher J (2018) Probing turbulent superstructures in rayleigh-bénard convection by lagrangian trajectory clusters. Physical Review Fluids 3:113501

Schröder A, Schanz D, Novara M, Philipp F, Geisler R, Knopp T, Schroll M, and Willert C (2018) Investigation of a high reynolds number turbulent boundary layer flow with adverse pressure gradients using piv and 2d-and 3d-shake-the-box. in 19th International Symposium on the Application of Laser and Imaging Techniques to Fluid Mechanics. 257

Sillero JA, Jiménez J, and Moser RD (2013) One-point statistics for turbulent wall-bounded flows at reynolds numbers up to $\delta^{+} \approx 2000$. Physics of Fluids 25:105102

Stasicki B, Schröder A, Boden F, and Ludwikowski K (2017) High-power led light sources for optical measurement systems operated in continuous and overdriven pulsed modes. in Optical Measurement Systems for Industrial Inspection X. volume 10329. page 103292J. International Society for Optics and Photonics

Wieneke B (2012) Iterative reconstruction of volumetric particle distribution. Measurement Science and Technology 24:024008

Zhang Q and Xiao Z (2018) Single-particle dispersion in compressible turbulence. Physics of Fluids 30:040904 